\title{
Gravity compensation in complex plasmas by application of a temperature gradient
}

\author{
H.Rothermel, T.Hagl, G.E.Morfill, M.H.Thoma, and H.M.Thomas \\ Centre for Interdisciplinary Plasma Science \\ Max Planck Institut für extraterrestrische Physik, Postfach 1312, D-85741 Garching, Germany
}

\begin{abstract}
Micron sized particles are suspended or even lifted up in a gas by thermophoresis. This allows the study of many processes occurring in strongly coupled complex plasmas at the kinetic level in a relatively stress-free environment. First results are presented. The technique is also of interest for technological applications.
\end{abstract}

Dusty or complex plasmas are low temperature, low pressure plasmas, e.g. glow discharge plasmas, containing microparticles in addition to ions, electrons and neutral gas. They have a large variety of important applications in fundamental and applied physics (see e.g. [1]). For example, the microparticles can, under certain conditions, form a crystal [2, 3, 4] which allows the study of the liquid-solid phase transition at the kinetic level [0]. Due to the large mass of the microparticles (typically $10^{13}$ times the mass of plasma ions) gravity is an important force. The normal procedure for complex plasma experiments under gravity conditions is to suspend the microparticles (which carry a negative charge, $Q$ ) in a strong electrostatic field, $E$, i.e. $m g=Q E+\sum_{i} F_{i}$, where the $F_{i}$ are other forces such as ion drag. To lowest order, $E$ varies linearly with position in a sheath of extent and it is easy to calculate that systematic body force variations from one lattice plane to the next exceed interparticle forces for the interesting particle size range above a few $\mu \mathrm{m}$. The systems are thus subject to a considerable amount of stress and there is abundant stored energy, which affects especially nonlinear processes such as phase transitions, interface dynamics and configurational or velocity space instabilities. It is for this reason that experiments under microgravity were proposed and are now being conducted [6.

It is the purpose of this paper to demonstrate that for monodisperse particles thermophoresis can be employed to counteract gravity, allowing complex plasma experiments to be performed in the "stress free" environment of the central nearly field-free plasma of a RF discharge, and to present first measurements. Results show that large stable systems can be produced in the charge neutral section of the plasma which exhibit the "central void" generally observed under $0-\mathrm{g}$ condition. New is the observed saturation of thermophoretic levitation in the case of a large particle number combined with low working pressure. Jellum et al. [7] were the first to apply a temperature gradient and to identify the thermophoretic effect in a dusty plasma. In order to compensate gravity, however, more prerequisites have to be fulfilled: a homogeneous temperature gradient by design, very low energy input to the gas, and the use of monodisperse particles.

Using elementary kinetic arguments, the thermophoretic force can be estimated similar to the heat conductivity [8]. The latter follows from the energy flux in the presence of a temperature gradient. Instead of the energy transfer, we consider the momentum transfer per unit time and area in a plasma. Then the thermophoretic force $F$ on a microparticle with radius $r_{p}$ is given as

$$
F=-\frac{8}{3} \frac{r_{p}^{2}}{v} \Lambda \frac{d T}{d x}
$$

where $v=(8 k T / \pi m)^{1 / 2}$ is the average thermal velocity of the gas atoms with mass $m$ at a gas temperature $T$ and $k$ is the Boltzmann constant. $\Lambda$ is the coefficient of heat conductivity and $d T / d x$ the temperature gradient.

As we will see, this formula provides very good results if measured values or the best theoretical calculations are used for $\Lambda$. The following result for mono atomic gases has been given in the literature [9]

$$
\Lambda=2.4 \frac{\eta c}{m}
$$

where $c=3 k / 2$ is the specific heat per atom and $\eta$ the shear viscosity of the gas. For the viscosity, we use a result derived from a precise solution of the transport problem in the case of hard spheres [8]

$$
\eta=0.553 \frac{\sqrt{m k T}}{\sigma}
$$

with the gas kinetic cross section $\sigma$ for atomic scattering, which can be taken from the literature 10, 11]. Using these cross sections, values for the viscosity of noble gases are obtained (see Table I) in good agreement with measured ones. For example, the measured viscosity of Argon at $T=300 \mathrm{~K}$ is $\eta=2.21 \cdot 10^{-5} \mathrm{Nsm}^{-2}$ compared to $2.16 \cdot 10^{-5} \mathrm{Nsm}^{-2}$ following from (3) together with the cross section $\sigma=4.2 \cdot 10^{-19} \mathrm{~m}^{2}$ given in Table I. Combining (11), (2), and (3) results in the thermophoretic force on a microparticle

$$
F=-3.33 \frac{k r_{p}^{2}}{\sigma} \frac{d T}{d x} .
$$

Like viscosity and heat conduction the thermophoretic force is pressure independent, as can been seen from (何) containing no pressure dependent parameters. Eq. (4) is valid only for spherical particles, mono atomic gases and 
low pressure where the mean free path is much larger than the particle radius.

We now compare our result to the literature. First, Waldmann [12] derived the thermophoretic force using the Enskog-Chapman method for solving the Boltzmann equation. He obtained the same expression as (1), where, however, the coefficient is given by $32 / 15$ instead of $8 / 3$, i.e., a thermophoretic force reduced by about $20 \%$ compared to our result.

In order to compare our result with the one by Havnes et al. [13, we use the relation $n \lambda=1 /(\sqrt{2} \sigma)$ [8] in (4), where $n$ is the number density of the gas and $\lambda$ the mean free path of the atoms, leading to

$$
F=-4.67 n k \lambda r_{p}^{2} \frac{d T}{d x}
$$

The result obtained by Havnes et al. follows from replacing the factor 4.67 by 8 in (5), i.e., they found a thermophoretic force which is larger than ours by almost a factor of two. Balabanov et al. [14 have used a formula for the thermophoretic force in a complex DC plasma which agrees with (5) assuming the ideal gas relation for the pressure and replacing the coefficient 4.67 by 4 .

TABLE I: Gaskinetic atom-atom cross-sections $\sigma$, mean free path $\lambda(p)$ at $50 \mathrm{~Pa}$, viscosity and heat conductivity at $293 \mathrm{~K}$, interaction radii $R=2 r=\sqrt{\sigma / \pi}$ from two sources. The thermophoretic force $F$ is compiled for the actual particle radius $r_{p}=1.69 \mu \mathrm{m}$ and temperature gradient of $1170 \mathrm{~K} / \mathrm{m}$. For a given temperature difference, $F$ is strongest for Helium.

\begin{tabular}{l|c|llllll} 
& Dim. & $\mathrm{He}$ & $\mathrm{Ne}$ & $\mathrm{Ar}$ & $\mathrm{Kr}$ & $\mathrm{Xe}$ & Ref. \\
\hline$\sigma$ & $10^{-20} \mathrm{~m}^{2}$ & 15 & 21 & 42 & 49 & 67 & 10 \\
$\lambda(p)$ & $10^{-4} \mathrm{~m}$ & 3.52 & 2.51 & 1.26 & 1.08 & 0.79 & \\
$\eta$ & $10^{-5} \mathrm{Nsm}^{-2}$ & 1.92 & 3.06 & $\mathbf{2 . 1 6}$ & 2.69 & 2.45 & (3) \\
$\Lambda$ & $10^{-2} \mathrm{Wm}^{-1} \mathrm{~K}^{-1}$ & 14.28 & 4.56 & $\mathbf{1 . 6 1}$ & 0.95 & 0.56 & \\
$R$ & $10^{-10} \mathrm{~m}$ & 2.19 & 2.59 & 3.66 & 3.95 & 4.62 & 10 \\
$R$ & $10^{-10} \mathrm{~m}$ & 2.18 & 2.56 & 3.66 & 4.14 & 4.88 & 11 \\
$F$ & $10^{-13} \mathrm{~N}$ & 10.2 & 7.31 & $\mathbf{3 . 6 6}$ & 3.13 & 2.29 & $\mathbb{1 1}$
\end{tabular}

Measurements were done in a completely symmetrical RF-excited plasma chamber with a volume of 400 $\mathrm{cm}^{3}$ (Fig. 1). The gas is heated from below and cooled from above by Peltier elements. The RF amplitude (with frequency $13.56 \mathrm{MHz}$ ) is applied to the electrodes with $180^{\circ}$ phase difference.

For calibration of the thermophoretic effect the discharge was operated at only $20 \mathrm{~mW}$. About $10^{4}$ particles were injected, much less than shown in e.g. Fig.2. The temperature difference between the metal plates ((4) in Fig.11) was increased until two narrow particle clouds, above and below the central void (Fig.3), of equal size were visible. For Argon gas the equilibrium was reached for temperatures of $54.6 \mathrm{C}$ at the lower electrode and $19.5 \mathrm{C}$ at the upper electrode. The temperature difference corresponds to a gradient of $1170 \mathrm{Km}^{-1}$.
As monodisperse particles still have a residual size and weight spectrum, particles observed above the void are different from those below in that they are about $5 \%$ less heavy. The 50/50 partition hence calibrates the thermophoretic force by the average particle weight. For the measured temperature gradient of $1170 \mathrm{Km}^{-1}$ (化) predicts a thermophoretic force of $3.66 \cdot 10^{-13} \mathrm{~N}$. Our particles (monodisperse melamine resin, diameter $3.4 \pm 0.1 \mu \mathrm{m}$, density $1510 \mathrm{~kg} / \mathrm{m}^{3}$ ) have a weight of $3.0 \pm 0.3 \cdot 10^{-13} \mathrm{~N}$. Thus, formula (14) is confirmed within the experimental uncertainties. We repeated the calibration with Neon and found equilibrium at a temperature difference of 17.7 K. Thus thermophoresis in Neon is twice as strong as in Argon as expected from the gas kinetic cross-section in Table 1 . We also verified the 50/50 partition as described above for a pressure range of 8 to $62 \mathrm{~Pa}$ and found no pressure dependence in thermophoresis as predicted by theory.

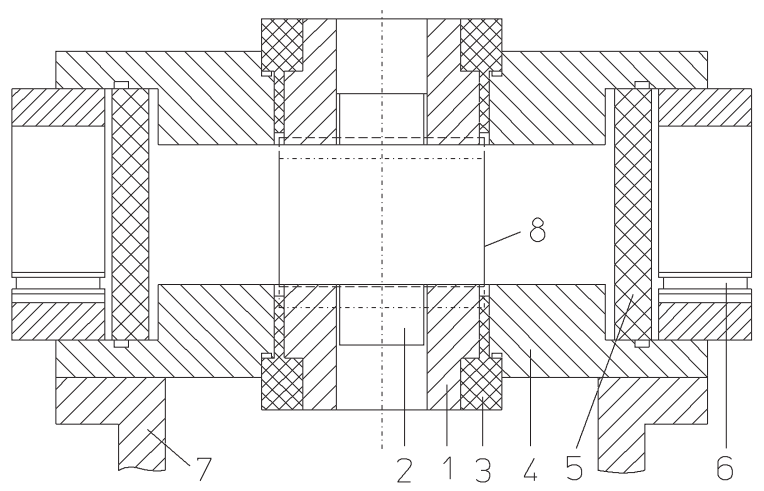

FIG. 1: Cross-section through the chamber: (1) stainless steel electrode, (2) dust dispenser, (3) Macor insulator, (4) metal plate at chassis potential, (5) window (welded Vycor body), (6) Peltier element, (7) heat sink, (8-10) fields of view (thermophoresis on: dashed line, off: dotted dashed). The distance between the electrodes is $30 \mathrm{~mm}$.

For comparison and further description of the apparatus we start with a condition where the Peltier elements and thermophoresis are not active (Fig. 2). The particle cloud settles down above the lower electrode due to gravity. One observes that the particles form a lattice, the socalled plasma crystal. The plasma glow was filtered away by an interference filter peaked on the laser wavelength of $686 \mathrm{~nm}$. Measurements taken without filter indicate the highest particle density congruent with the brightest glow. There is no convection after the particles have arranged themselves into the ordered structure. The laser cut is focused to $\approx 50 \mu \mathrm{m}$ full width, much narrower than the lattice spacing. The stringlike structures, caused by the wake potential of the positive ions streaming to the lower electrode, disappear in patches, corresponding to local distortions no longer illuminated. The three dimensional structure of such a system was analyzed by Zuzic et al. 15. The RF power measured at the genera- 
tor is $170 \mathrm{~mW}$, the peak to peak amplitude measured by a voltage probe at the electrode is $45 \mathrm{~V}$. Both electrodes are shunted with $3.3 \mathrm{k} \Omega$ to chassis. Losses in the unbalanced/balanced transformer, the matching circuits and in particular the shunt resistors dictate that only $10 \%$ (17 $\mathrm{mW}$ ) of the RF power go to the discharge.

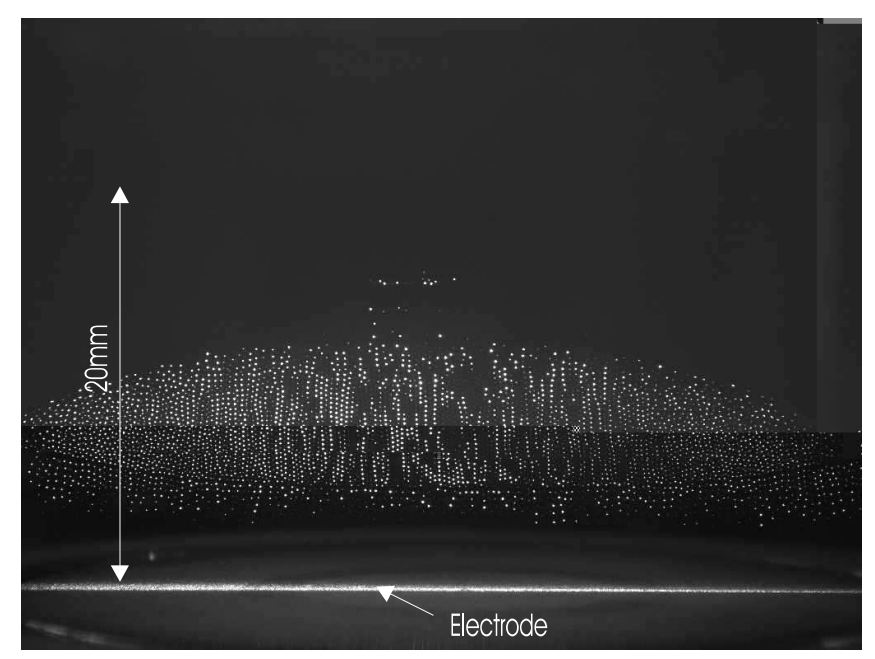

FIG. 2: $\quad$ Side view of $3.4 \mu \mathrm{m}$ microparticles suspended in a plasma (complex plasma), when no external temperature gradient is present. The gas is Argon, the pressure $48 \mathrm{~Pa}$, the $\mathrm{RF}$ amplitude $45 \mathrm{~V}$ peak to peak, the discharge power $17 \mathrm{~mW}$. The number of particles is about $10^{6}$, the field of view $32 \times 43 \mathrm{~mm}$. The lowest 5 lattice planes of large interparticle distance consist of agglomerates.

For the thermophoretic measurements the predetermined gradient of $1170 \mathrm{~K} / \mathrm{m}$ was applied, the plasma was started and the discharge power was set to a medium value of $20 \mathrm{~mW}$. Particles were injected until the plasma appeared to be saturated. To accomplish a stable discharge in the quasi 0-g case, higher RF amplitudes and powers are required. A survey was measured at three power levels $(40,57,72 \mathrm{~mW})$ and four pressures $(14,24$, 38, $46 \mathrm{~Pa})$.

Fig. 3 and 4 are taken out of the survey in order to visualize the stress-free complex plasma under quasi $0-\mathrm{g}$ and in order to show a saturation of thermophoresis in the presence of a large particle number combined with a small working pressure. Fig. 3 shows how the particle cloud fills the whole discharge volume apart from space charge regions near to the electrodes and the central region where a void is formed [6]. The reason for no lattice structure in Fig. 3 could be a residual convective motion in the order of $1 \mathrm{~mm} / \mathrm{s}$ which is not visible in a single video frame. The void boundary appears well defined with a slight enhancement of particle density behind the boundary. Using $\Lambda$ as given in table 1 we calculate a total heat flow from the lower to the upper electrode of $24 \mathrm{~mW}$. On the other hand the RF power dissipated in the plasma is roughly $57 \mathrm{~mW}$. Most of this power will be dissipated by ionisation and heating of the electrodes by ion impact. Only a few $\mathrm{mW}$ will heat the neutral gas directly, hence we expect that the temperature profile applied from outside for thermophoresis remains reasonably homogeneous for the discharge parameters used, although a small amount of Joule heating in the center of the chamber might contribute to the void formation. If we compare our experimental condition to the one reported by Jellum et al. [7], we find from the amplitudes and the power levels that they drive their discharge at 5 to 10 times the power density that we use. If we increase the discharge power by 10 , we find that the particles are driven out of the bulk plasma by both the ion drag force and intrinsic thermophoresis.

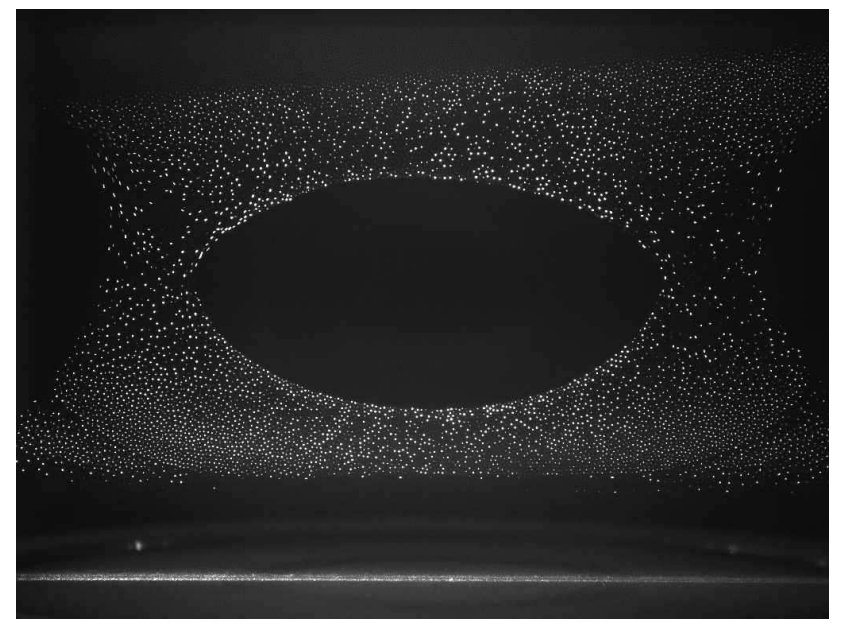

FIG. 3: Side view of a complex plasma at quasi $0-\mathrm{g}$ accomplished by thermophoresis. The temperature gradient applied from outside is $1170 \mathrm{~K} / \mathrm{m}$. The peak to peak amplitude is $82 \mathrm{~V}$, the discharge power $57 \mathrm{~mW}$, the pressure $46 \mathrm{~Pa}$ and the number of particles injected in the order of 1 million.

Obviously thermophoresis will not support an unreasonably large total mass of particles. A saturation in the thermophoretic force becomes visible first at low working pressure. Fig. A is a measurement under identical conditions as Fig. 3, however, at a working pressure of $14 \mathrm{~Pa}$. The particles settle down towards the lower electrode. This effect can be reversed by increasing the pressure again to $46 \mathrm{~Pa}$. Out of a number of possible mechanisms our data as well as rough estimates identify radiation cooling (Brattli and Havnes [16]) as the best explanation. By thermal radiation the particles will approach everywhere a temperature roughly equal to the average temperature of the chamber surfaces. Hence for a large particle density and good thermal coupling between the particles and the gas the temperature gradient in the dust region will be reduced and the gradient in the dust free region will become correspondingly larger. A reduced temperature gradient in the dust region weakens the thermophoretic force. Gravity gains the overhand and the dust settles down towards the lower (heated) 
electrode as observed. At larger pressure the decreasing mean free path of the neutral atoms reduces the thermal coupling between the gas and the particles, causing the reversal of this effect. A quantitative understanding of this effect requires detailed investigations beyond the scope of the present Letter.

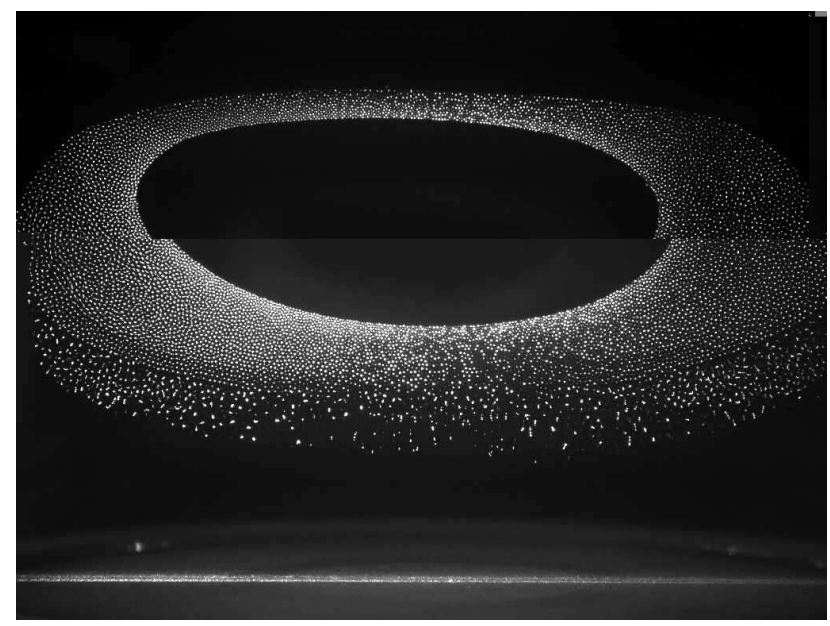

FIG. 4: Side view under identical conditions as Fig. 3 with only the gas pressure reduced from $46 \mathrm{~Pa}$ to $14 \mathrm{~Pa}$. As the thermophoretic force becomes saturated particles settle down below the void. Interesting is a strong enhancement of particle density behind a very sharp void boundary.

At ambient pressure $\left(10^{5} \mathrm{~Pa}\right)$ gravity driven convection would obstruct thermophoresis completely. At working pressures between 46 and $14 \mathrm{~Pa}$ convection is 3 orders of magnitude weaker. The driving force of vorticity depends on the temperature difference, the free fall acceleration and the mass density of the gas. The driving force hence decreases in proportion to pressure whereas the counteracting friction on the chamber walls remains unchanged as the viscosity of gases is pressure independent.

In conclusion, the thermophoretic levitation of microparticles in a complex plasma, can give interesting insights into complex plasmas and be used as a promising tool complementary to microgravity experiments. As an example we discussed radiation cooling by the particles, leading to the observed particle density dependent reduction of the temperature gradient. Other important exampels are the investigation of the void and vortices formation, also observed under microgravity conditions. Furthermore we present an easy to use relation for the thermophoretic force together with the relevant gas kinetic data. Technological applications of thermophoresis could be size selective particle production in a reactive plasma and surface plating of particles. In semiconductor production where nanometer sized particles are a byproduct in some processes, controlled thermophoresis may be used to keep particles away from the substrate where they can diminish the yield, as already suggested by Jellum et al. [7], or to incorporate them in a controlled way where they are beneficial, e.g. for amorphous solar cells [17. Controlled thermophoresis could be interesting for "pick and place" in context with hybrid integration [18].

\section{Acknowledgements}

The work was triggered by a microgravity project funded by: DLR (BMBF), grant no. 50WB9852. We have to thank Prof. O. Havnes (University of Troms $\varnothing$ ) and our colleagues U. Konopka, K. Tarantik and M. Zuzic for valuable suggestions and help with the manuscript.

[1] A. Bouchoule (Ed.), Dusty Plasmas (John Wiley, Chichester 1999).

[2] J.H. Chu and Lin I, Phys. Rev. Lett. 72, 4009 (1994).

[3] H.M. Thomas et al., Phys. Rev. Lett. 73, 652 (1994).

[4] Y. Hayashi and K. Tachibana, Jpn. J. Appl. Phys. Part 1 33, 804 (1994).

[5] H.M. Thomas and G.E. Morfill, Nature 379, 806 (1996).

[6] G.E. Morfill, H.M. Thomas, U. Konopka, H. Rothermel, M. Zuzic, A. Ivlev, and J. Goree, Phys. Rev. Lett. 83, 1598 (1999).

[7] G.M. Jellum, J.E. Daugherty, and D.B. Graves, J. Appl. Phys. 69, 6923 (1991).

[8] F. Reif, Fundamentals of Statistical and Thermal Physics (McGraw-Hill, New York 1965).

[9] C. Gerthsen, Physik (Springer, Berlin 1958).

[10] R.N. Varney, Phys. Rev. 88, 362 (1952).

[11] M. Wutz, H.Adam, and W. Walcher, Theorie und Praxis der Vakuumtechnik (Vieweg, Braunschweig 1981).

[12] L. Waldmann, Z. Naturforsch. 14a, 259 (1959).

[13] O. Havnes, T. Nitter, V. Tsytovich, G.E. Morfill, and T. Hartquist, Plasma Sources Sci. Technol. 3, 448 (1994).

[14] V.V. Balabanov et al., JETP 92, 86 (2001).

[15] M. Zuzic, A. Ivlev, J. Goree, G.E. Morfill, H.M. Thomas, H. Rothermel, U. Konopka, R. Sütterlin, and D.D. Goldbeck, Phys. Rev. Lett. 85, 4064 (2000).

[16] A. Brattli and O. Havnes, J. Vac. Sci. Technol A, 14, 644 (1996).

[17] P. Rocca i Cabarroccas, P. Stahel, S. Hamma, and Y. Poissant, $2^{\text {nd }}$ World conference and exhibition on photovoltaic solar energy conversion, (Vienna, 1998), 355.

[18] J. Wallace: Hybrid Integration: Light positions optoelectric parts, Laser Focus World, Dec. 2000, 30. 